\title{
Measurements of the Propagation Parameters of Tree Canopies at MMW Frequencies
}

\author{
A. Y. Nashashibi, F.T. Ulaby, P. Frantzis, and Roger D. De Roo \\ The Radiation Laboratory \\ Department of Electrical Engineering and Computer Science \\ The University of Michigan, Ann Arbor, MI 48109-2122
}

\begin{abstract}
The presence of trees in a given scene can hamper detection of nearby targets by millimeter-wave radars especially at near grazing incidence. Proper characterization of scattering and attenuation in tree canopies is important for optimal detection algorithms. In this paper, a new technique for determining the extinction and volume backscattering coefficients in tree canopies using the measured radar backscatter response is proposed and verified experimentally. The technique, which can be applied to already available wideband radar backscatter data, is used to compute the extinction and volume backscattering coefficients of different tree canopies under various physical conditions. The dynamic range of these coefficients are presented and results at $35 \mathrm{GHz}$ are compared with results at $95 \mathrm{GHz}$.
\end{abstract}

* Prepared through collaborative participation in the Advanced Sensors Consortium sponsored by the U.S. Army Research Laboratory under the Federated Laboratory Program, Cooperative Agreement DAAL01-96-2-0001. 


\section{INTRODUCTION}

The advantages of millimeter-wave (MMW) radars over other sensors include high resolution, near all weather capabilities, light weight, and compactness. These advantages have motivated a number of research efforts on MMW radar phenomenology over the past two decades. Both experimental and theoretical modeling efforts aimed at characterizing the radar response of different types of clutter were reported [1]- [7]. With recent advancements in semiconductor technologies, reliable and affordable MMW radars are now attainable. They have become favorable sensors for many civilian and military applications, especially at near grazing incidence. A multi-year research program has been established at The University of Michigan to characterize the MMW radar backscatter response of clutter at near grazing incidence where little research is reported in the literature.

The presence of tree canopies, the focus of this paper, in a radar scene could potentially influence the ability of a MMW radar in detecting targets at near grazing incidence. Due to the substantial physical extent of trees above the ground surface, they not only possess a significant radar backscatter cross section, but they can also hamper the detection of targets positioned behind them. In a tenuous random medium, such as a tree canopy, whose scatterers are lossy and often several wavelengths in dimension (at MMW frequencies), signal propagation and scattering inside the medium can be expressed in terms of two quantities, the extinction coefficient, $\kappa_{e}$, measured in units of $\mathrm{Np} / \mathrm{m}$, and the backscattering cross section per unit volume, $\sigma_{v}$, measured in units of $\mathrm{m}^{2} / \mathrm{m}^{3}$ and often called the volume backscattering coefficient [13]. In general, the values of $\kappa_{e}$ and $\sigma_{v}$ depend on both the physical parameters characterizing the tree canopy, such as leaf size and orientation distributions, gravimetric moisture content, number density, etc., and on the radar parameters, such as frequency, local incidence angle, and polarization. Proper characterization of $\kappa_{e}$ and $\sigma_{v}$ in terms of both the canopy's physical parameters and the radar parameters is useful in model calculations of the total backscatter from and penetration through the tree. In addition, they can aid in the development of radar image simulations of forested terrain and in the design of the optimum sensor, as well as other applications.

A number of research efforts aimed at characterizing propagation and bistatic scattering in tree canopies have 
been reported in the past [4]- [7]. They demonstrated degradation in coherence and amplitude as MMW signals propagate in forest stands. However, due to the inherent difficulties in the experimental techniques used, these studies were limited to few types of trees and/or observations over portions of the growth season. In this paper, we report on an ongoing effort to characterize $\kappa_{e}$ and $\sigma_{v}$ for different types of tree canopies. In the next section, a new technique for determining $\kappa_{e}$ and $\sigma_{v}$ of tree canopies using the measured backscatter response is presented. Then, the technique is verified experimentally in a laboratory environment. Finally, examples of recent experimental observations and results are discussed.

\section{NEW TECHNIQUE FOR MEASURING EXTINCTION IN TREE CANOPIES}

Traditionally, extinction measurements of random media were conducted using the free-space transmission measurement technique (FSTM) [8], [9]. In this standard approach, the random medium is positioned between the transmit and receive antennas and the field propagating through the medium is measured over many independent realizations of the medium. The field detected by the receive antenna $E^{r}$ consists of two components, an incoherent component due to scattering in the forward direction and a coherent component. The coherent component is the incident field $E^{i}$ partially reduced in amplitude due to scattering and absorption in the random medium.

It is straightforward to show that by averaging the received field coherently over many independent realizations of the same random medium, an explicit expression relating the power of the mean received field $P^{r}$ to the incident power can be derived:

$$
P^{r}=\frac{1}{2 \eta}\left|<E^{r}>\right|^{2}=P^{i} e^{\left(-\kappa_{e} d\right)}
$$

where $P^{i}=\frac{1}{2 \eta}\left|E^{i}\right|^{2}$ is the incident power, $\kappa_{e}$ is the extinction coefficient (in $\mathrm{Np} / \mathrm{m}$ ), $d$ is the thickness of the random-medium layer, and $\eta$ is the intrinsic impedance. With this technique, $\kappa_{e}$ of a random medium can be easily determined in a laboratory environment. However, it becomes practically impossible to perform on tree canopies outdoors, especially at MMW frequencies where the requirements for both signal coherence and antenna alignment are difficult to maintain. 
In this section, we propose an alternative technique in which the extinction coefficient of a tree canopy is determined from radar backscatter measurements. This is particularly advantageous, because wide-band polarimetric data originally acquired for the purpose of characterizing the polarimetric radar backscatter response of a tree canopy (the backscattering coefficients) can be used to extract the value of $\kappa_{e}$ of the same canopy. To first order, a tree canopy can be viewed as a tenuous layer of randomly oriented and lossy scatterers (foliage) embedded in lossless background with diffuse air-canopy interface. It can be shown that in the backscatter configuration, depicted in Fig. 1, the mean backscattered power $P_{b}^{r}$ from a given depth $z$ in the tree canopy can be expressed in terms of the incident power,

$$
P_{b}^{r}(z)=\frac{1}{2 \eta}<\left|E_{b}^{r}\right|^{2}>=P^{i} \sigma_{v} e^{-2 \kappa_{e}\left(z-z_{o}\right)},
$$

where $\sigma_{v}$ is the volume backscattering coefficient. In equation (2), effects of multiple scattering were implicitly neglected. If, in addition, the foliage is assumed to be uniformly distributed inside the canopy, then both $\kappa_{e}$ and $\sigma_{v}$ become independent of position and can be determined from the measured backscatter responses from at least two different depths in the tree canopy. The response from multiple depths in the canopy can be acquired through the use of wide-band radars. This in turn can improve the determination of $\kappa_{e}$ and $\sigma_{v}$.

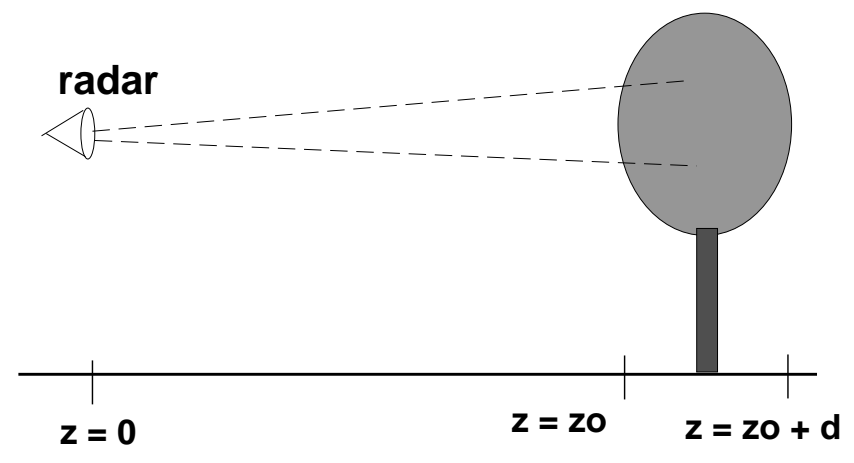

Figure 1: Proposed experimental setup to measure the extinction of a tree canopy from the mean backscatter response.

In practice, both the radar system antenna patterns and the shape of the radar pulse incident on the tree canopy influence the backscatter response. Hence, they must be removed prior to the determination of $\kappa_{e}$ and $\sigma_{v}$. 


\subsection{Mathematical Derivation:}

To begin our examination of the mathematical formulation characterizing the time-domain radar backscatter response of a distributed medium such as a tree canopy, let us first consider the response due to a point target. The power received from a point target with radar cross section $\sigma_{p}$ and positioned at a distance $R_{p}$ away from a $\mathrm{CW}$ radar is

$$
P_{b}^{r}=\frac{P^{t} G_{t 0} G_{r 0} \lambda^{2}}{(4 \pi)^{3} R_{p}^{4}} \sigma_{p}
$$

where $P^{t}$ is the transmitted power, $G_{t 0}$ and $G_{r 0}$ are the transmit and receive antenna gains respectively, and $\lambda$ is the wavelength. In the case of a wide-band radar, the received power is the result of convolving the transmitted pulse, $I(t)$, with the impulse response of the given target, $h(t)$ (i.e., $P_{b}^{r}(t)=I(t) * h(t)$ ). For a point target positioned at $R_{p}$ (with $t_{p}=2 R_{p} / c$, where $c$ is the speed of light), its impulse response can be expressed as $h\left(t-t_{p}\right)=\sigma_{p} \delta\left(t-t_{p}\right)$ and the received power becomes

$$
\begin{aligned}
P_{b}^{r}(t) & =\frac{K}{R_{p}^{4}} \sigma_{p} \int I(t-\tau) \delta\left(\tau-\frac{2 R_{p}}{c}\right) d \tau \\
& =\frac{K}{R_{p}^{4}} \sigma_{p} I\left(t-\frac{2 R_{p}}{c}\right),
\end{aligned}
$$

where $K=P^{t} G_{t 0} G_{r 0} \lambda^{2} /(4 \pi)^{3}$. Hence, by measuring a point target with known RCS, such as a metallic sphere, both the shape of the pulse being transmitted and the value of $K$ may be determined.

Example: For a Gaussian pulse $I(t)=e^{-a t^{2}}$, the expression for the received power reduces to

$$
P_{b}^{r}(t)=\frac{K}{R_{p}^{4}} \sigma_{p} e^{-a\left(t-2 R_{p} / c\right)^{2}}
$$

For a distributed target, such as a tree canopy, the radar antenna system illuminates a portion of the target as depicted in Fig. 1. In this case, the antenna patterns for both transmit and receive antennas, defined as $G_{t}(\theta, \phi)=G_{t 0} g_{t}(\theta, \phi)$ and $G_{r}(\theta, \phi)=G_{r 0} g_{r}(\theta, \phi)$, need to be included. Assuming that the foliage is uniformly distributed inside the canopy, i.e. $\kappa_{e}$ and $\sigma_{v}$ are constant and independent of depth, then the contribution of a 
slice inside the tree canopy of width $d z^{\prime}$ to the received power in range bin $z=(c t / 2)$, due to a Gaussian pulse $I(z)=e^{-a(2 z / c)^{2}}$ (considered here for simplicity), is given by

$$
\begin{aligned}
d P_{b}^{r}(z)= & K \sigma_{v} e^{-a\left(2\left(z-z^{\prime}\right) / c\right)^{2}} e^{-2 \kappa_{e}\left(z^{\prime}-z_{0}\right)} \\
& \iint_{\text {illum.area }} \frac{g_{t}(\theta, \phi) g_{r}(\theta, \phi)}{\left(z^{\prime}\right)^{4}} d A d z^{\prime}
\end{aligned}
$$

In case of a high resolution radar, the illuminated area $A$ can be assumed constant over the resolution cell and $z^{\prime}$ inside the integral can be approximated with $z$. As a result, the total received power at a given range $z$ (with $t=2 z / c)$ can be computed from

$$
P_{b}^{r}(z)=K A \sigma_{v} \int_{z^{\prime}=z_{0}}^{z_{0}+d} e^{-a\left(2\left(z-z^{\prime}\right) / c\right)^{2}} e^{-2 \kappa_{e}\left(z^{\prime}-z_{0}\right)} d z^{\prime}
$$

where $d$ is the thickness of the canopy, and $A$ is the effective cross sectional area of the radar beam,

$$
A=\iint_{\text {illum.area }} \frac{g_{t}(\theta, \phi) g_{r}(\theta, \phi)}{\left(z^{\prime}\right)^{4}} d A
$$

In equation (7), the mean backscattered power due to a Gaussian pulse propagating through a tree canopy (consisting of a homogeneous set of scatterers) is expressed in terms of $\kappa_{e}$ and $\sigma_{v}$. These two quantities can be determined from the measured backscatter response of a tree canopy by minimizing the difference between the measured response and the theoretically calculated response in (7); that is:

$$
\text { minimize }\left|P_{\text {theory }}^{r}(z)-<P_{\text {measured }}^{r}(z)>\right|^{2} \quad \text { over } z_{0}<z<z_{0}+d \text {. }
$$

\subsection{Numerical Simulations:}

The influence that the pulse shape and extinction coefficient have on the expected time-domain response of a random medium, can be best characterized through numerical simulations. For simplicity, we considered a layer of tenuous and homogeneous random medium of thickness $d$ consisting of identical scatterers. In addition, assuming plane-wave incidence and setting both the magnitude of the incident field and the effective cross 
sectional area of the radar beam $A$ to one, the backscattered electric field at a given frequency is computed by summing coherently the fields from all scatterers within the layer:

$$
E_{b}^{r}(f)=\sum_{l=1}^{N} \frac{e^{-2 i k z_{l}}}{z_{l}} S_{o} E^{i}(f),
$$

where $f$ is the frequency, $N$ is the number of scatterers in the medium, $z_{l}=z_{o}+z_{l}^{\prime}\left(\right.$ with $0<z_{l}^{\prime}<d$ ) is the range to the $l$ th scatterer, $k=\beta-i \kappa_{e} / 2$ is the wave number (with $\beta=2 \pi f / \mathrm{c}$ ), and $S_{o}$ is the scattering amplitude of the the identical scatterers. Both $E^{i}(f)$ and $S_{o}$ are set to one for convenience. Since extinction occurs inside the random medium only, equation (10) can be reduced to

$$
E_{b}^{r}(f)=\sum_{l=1}^{N} \frac{e^{-2 i \beta z_{l}}}{z_{l}} e^{-\kappa_{e}\left(z_{l}-z_{o}\right)} .
$$

Simulations of the time domain radar backscatter response can be conducted as follows: (1) generate the scatterer positions within the medium using a random number generator, (2) compute the backscattered fields $E_{b}^{r}$ over $M$ frequency points spanning the desired bandwidth, and (3) perform an inverse Fourier transform on $E_{b}^{r}(f)$ to produce the time-domain response $e_{b}^{r}(t)$ (hence, $\left.P_{b}^{r}(t)=\frac{1}{2 \eta}\left|e_{b}^{r}(t)\right|^{2}\right)$. By repeating steps 1 through 3 for many realizations of the random medium, the mean backscattered power can then be obtained from the timedomain response averaged over all realizations. The procedure follows closely the data acquisition and signal processing technique employed in the next section where a network analyzer-based radar (stepped-frequency measurements) is used to verify experimentally the new technique.

The simulated responses of a 3-m thick layer consisting of 3000 randomly located scatterers are plotted in Figs. 2 and 3. These simulations were performed over 1-GHz bandwidth (0.15 m resolution), in which 100 independent realizations of the random medium were considered. In Fig. 2 the sensitivity of the backscatter response, especially its rate of decay in the time-domain, to $\kappa_{e}$ is clearly apparent. In Fig. 3, the simulated response is compared with those predicted by (7) assuming in one case a Gaussian pulse and in the other a Sinc-square pulse $\left(|\sin x / x|^{2}\right)$ while setting $A=\sigma_{v}=K=1$ for simplicity. Excellent agreement is observed between the simulated response and those based on (7). This indicates that the Gaussian pulse model can be used in computing $\kappa_{e}$ and $\sigma_{v}$, irrespective of the modulation used (whether stepped frequency or pulse) as long as the 
bandwidth is the same. This can be easily insured by selecting a Gaussian pulse-width such that the expression in (5) provides a time-domain response that matches the response measured for an actual point target.

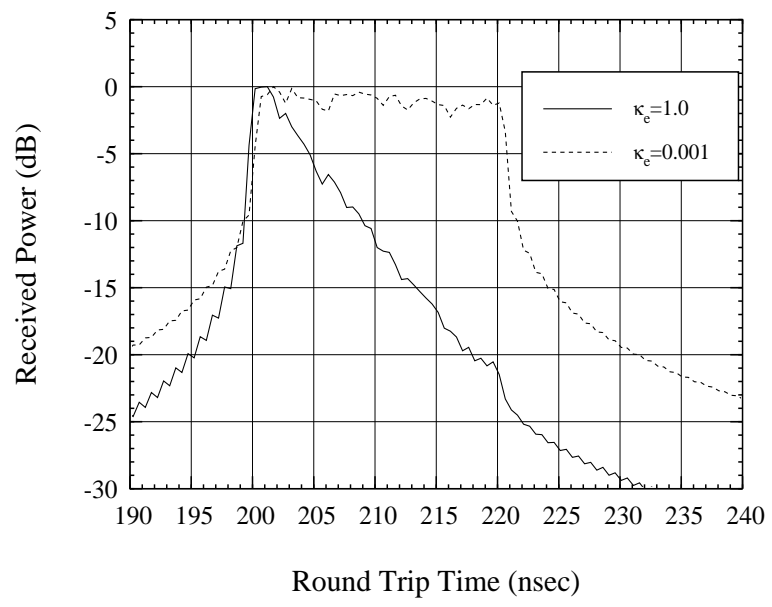

Figure 2: The effect of the extinction coefficient on the time-domain radar backscatter response of a homogeneous random medium. Numerical simulations of 100 independent samples were conducted assuming $z_{o}=30 \mathrm{~m}$, $d=3 \mathrm{~m}, N=3000$, and 1.0-GHz bandwidth.

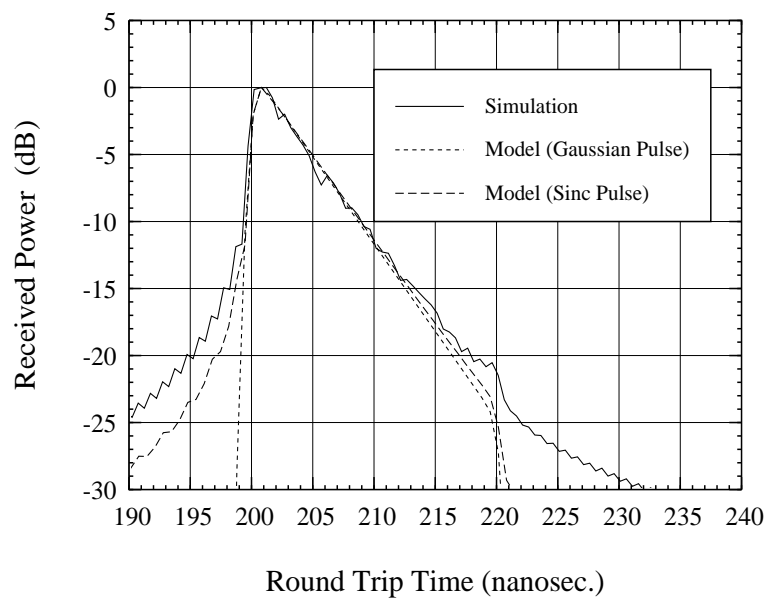

Figure 3: The effect of the pulse shape on the time-domain radar backscatter response of a homogeneous random medium. Numerical simulations of 100 independent samples were conducted assuming $z_{o}=30 \mathrm{~m}, d=3 \mathrm{~m}$, $N=3000, \kappa_{e}=1.0$, and 1.0-GHz bandwidth. 


\section{EXPERIMENTAL VERIFICATION}

The applicability of the new technique to the measurement of extinction in tree canopies at MMW frequencies was verified experimentally by conducting two sets of indoor measurements on an Arbor Vitae tree. A fully polarimetric network analyzer-based Ka-band instrumentation radar was used [11,12]. The dual antenna radar (with $2.4^{\circ}$ effective beamwidth) was operated over 1.0-GHz of bandwidth centered around $34.5 \mathrm{GHz}$. In all measurements, the vv and hh-polarized responses of 50 independent spatial samples were collected by rotating the tree about its axis. Tree rotation was facilitated using a computer-controlled stepper motor. In all measurements, the effective antenna spot size was substantially smaller than the tree diameter, insuring a distributed target-type measurement.

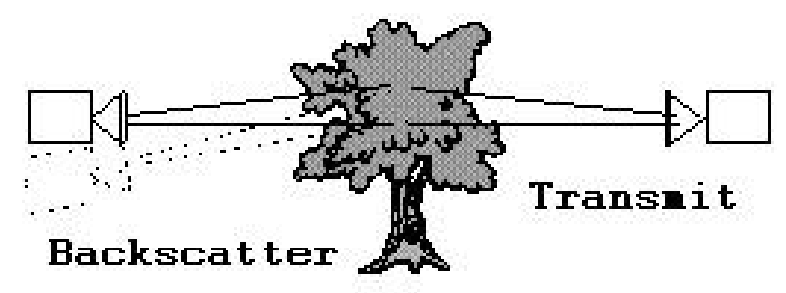

Figure 4: Indoor experimental setup demonstrating both transmit-through and backscatter modes of operation of the Ka-band radar.

The radar was first operated in a transmit-through mode. In this mode, the two modules comprising the radar's $\mathrm{RF}$ front-end, the transmit and receive modules, were separated and positioned with the antennas pointed at each other in a transmit-through arrangement as depicted in Fig. 4. The tree was then positioned half-way between the two modules while at the same time maintaining the far field requirements for both antennas. Coherence between the two modules was maintained by injection-locking the transmit and receive local oscillators with the third harmonic of an X-band signal. The system was calibrated using the free space (no tree) measured response. Then, the extinction coefficients for both vertically and horizontally polarized waves were extracted from the measured response following the expression in (1). Their values were $\kappa_{e}^{v}=3.8 \mathrm{~Np} / \mathrm{m}$ and $\kappa_{e}^{h}=3.6 \mathrm{~Np} / \mathrm{m}$, respectively. 
In the second measurement, the radar was operated in the backscattering mode. A 2-inch metallic sphere was measured and its polarimetric response was used in the calibration technique described by Nashashibi et al. [12]. Equations (7) and (9) were used to extract the extinction coefficients from the backscattered response. Excellent agreement was obtained between the extinction coefficients measured using both the transmit-through technique and the new proposed technique for both vv- and hh-polarized cases. In Fig. 5, both the averaged radar backscatter response of the Arbor Vitae tree canopy and the model-based response using $\kappa_{e}^{v}=3.8 \mathrm{~Np} / \mathrm{m}$ are plotted as a function of the round trip propagation time. The figure demonstrates that the new measurement technique is capable of measuring the extinction coefficients accurately.

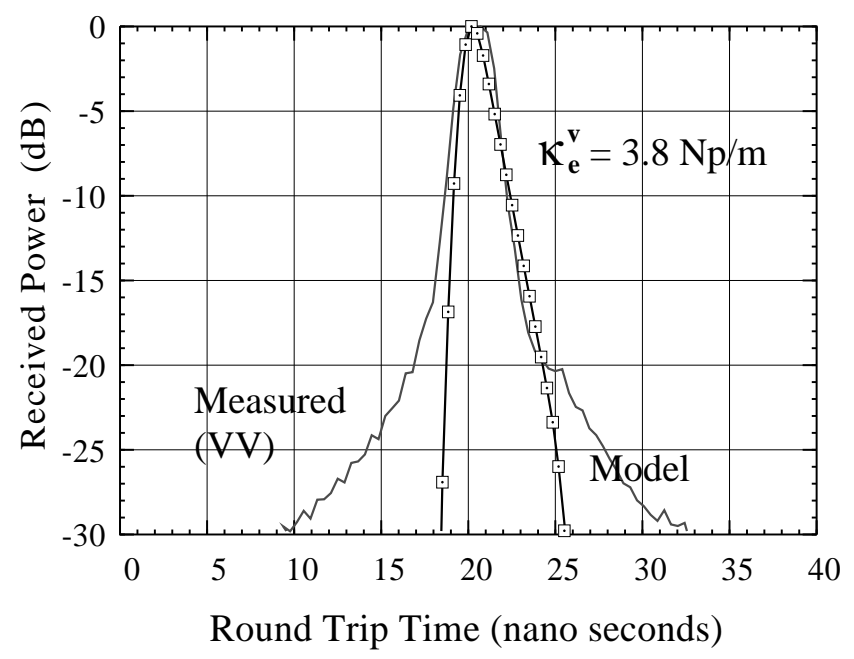

Figure 5: Comparison between the mean backscatter response of an Arbor Vitae tree canopy, measured at 35 $\mathrm{GHz}$ in an indoors setting, and the model-based response in (7). The value of $\kappa_{e}^{v}$ used in the model is the same one derived from transmit through measurements.

\section{EXPERIMENTAL OBSERVATIONS AND PRELIMINARY RESULTS}

Over the past two years, The University of Michigan has embarked on an extensive outdoor measurement campaign of the MMW polarimetric radar response of clutter at near grazing incidence. In these measurements, two ultra-fast, coherent, wide-band (500 MHz), fully polarimetric instrumentation radars operating at 35 and 95 $\mathrm{GHz}$ were employed to characterize the polarimetric radar backscatter response of more than 50 tree canopies. 
Hundreds of data sets were collected, in which most canopies were measured several times during their growth cycles. At least 80 spatially independent samples of the tree canopy were measured at any given time by pointing the radar beam at different areas of the canopy. Along with the radar measurements, physical parameters characterizing the tree canopies were collected and compiled. Table 1 contains a partial listing of the physical parameters collected for a number of tree canopies examined in this paper.

In addition to the standard processing techniques employed on the measured data in order to compute the modified Mueller matrices of the tree canopies [10]- [12], the technique described in the previous section was used to compute $\kappa_{e}$ and $\sigma_{v}$ from the incoherently averaged time-domain response. It was observed that whenever foliage in the tree canopy was not close to being uniformly distributed throughout the canopy, such as was the case of a de-foliated Norway Maple tree, then the time-domain response was rather complex and the model discussed in the previous section could not be used. In addition, it was observed that the number of leaves $/ \mathrm{m}^{3}$ or fruits $/ \mathrm{m}^{3}$ and the structure of the tree seem to be the dominant parameters influencing the variations in $\kappa_{e}$ and $\sigma_{v}$. On the other hand, slight variations in the gravimetric moisture content, $m_{g}$, and leaf area for a given tree canopy had no noticeable influence on the measured radar response of the canopy.

The extinction coefficient of an Arbor Vitae (an evergreen tree) measured at different times is plotted in Fig. 6. The magnitude of the extinction coefficient of the canopy is twice as large during late Summer and through the Fall in comparison with Spring and early Summer. This can be attributed to the presence of fruits (conical in shape) in the tree canopy late in the growth period. In addition, extinction tends to be slightly higher at $95 \mathrm{GHz}$ when compared to $35 \mathrm{GHz}$.

The extinction coefficients of the trees listed in Table 1 are summarized in Fig. 7. The figure clearly demonstrates the wide variations in $\kappa_{e}$ that exist across tree species, in general, and within a given tree species in particular. These variations intensify further when the total attenuation through the whole canopy is considered. In Fig. 8, at least $50 \mathrm{~dB}$ of dynamic range in total attenuation is observed between the different types of canopies.

A wide dynamic range is also observed for the volume backscattering coefficients, as shown in Fig. 9. However, 


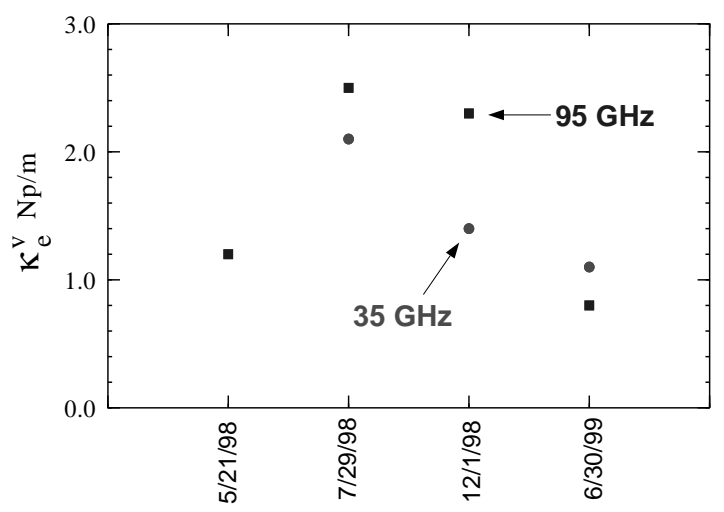

Figure 6: Sensitivity of the extinction coefficient to changes in the life cycle of an Arbor Vitae tree. During the Fall, the tree canopy bore fruits.

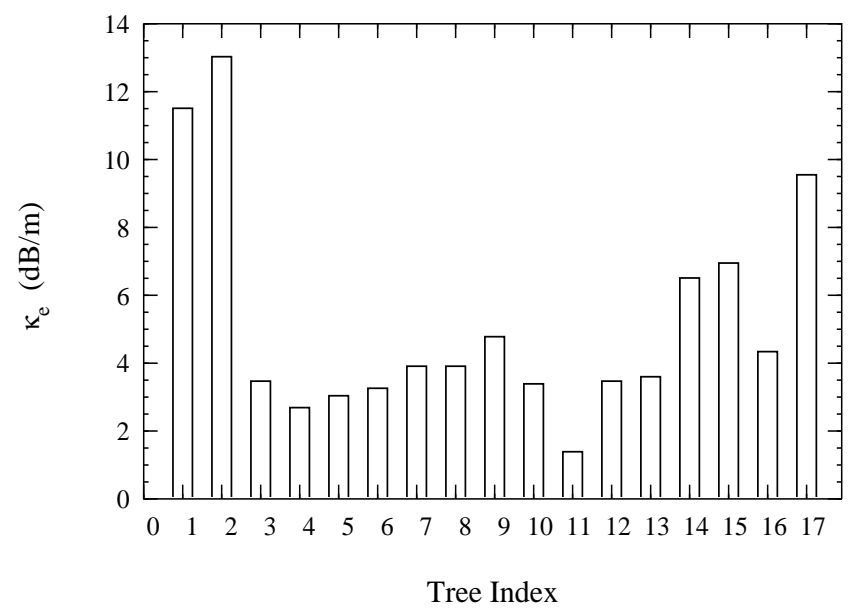

Figure 7: Dynamic range of the extinction coefficients $(\mathrm{dB} / \mathrm{m})$ computed at $95 \mathrm{GHz}$ for the trees listed in Table 1. no significant correlation between the values of $\kappa_{e}$ and $\sigma_{v}$, plotted against each other in Fig. 10, is observed. It can be shown that the backscattering coefficient $\sigma^{o}\left(\mathrm{~m}^{2} / \mathrm{m}^{2}\right)$, which represents the total backscatter response per unit area, can be related to $\kappa_{e}$ and $\sigma_{v}$ of the canopy through the following radiative transfer-based equation, [13],

$$
\sigma^{o}=\frac{\sigma_{v}}{2 \kappa_{e}}\left(1-e^{-2 \kappa_{e} d}\right)
$$

The modeled $\sigma_{v v}^{o}$ (computed using (12)) is compared in Fig. 11 with the measured $\sigma_{v v}^{o}$. The plot indicates that our derived values of $\kappa_{e}$ and $\sigma_{v}$ are in very good agreement with the measured data. Less than $30 \%$ of the data points show a difference between the measured and modeled $\sigma_{v v}^{o}$ values that exceeds $1 \mathrm{~dB}$. In fact, for these data 


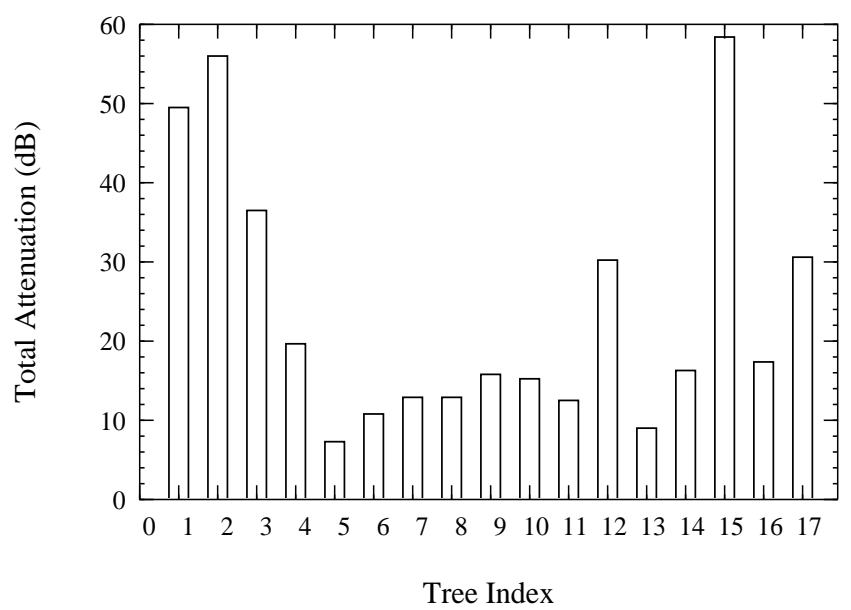

Figure 8: Dynamic range of the total attenuation through the canopy computed at $95 \mathrm{GHz}$ for the trees listed in Table 1.

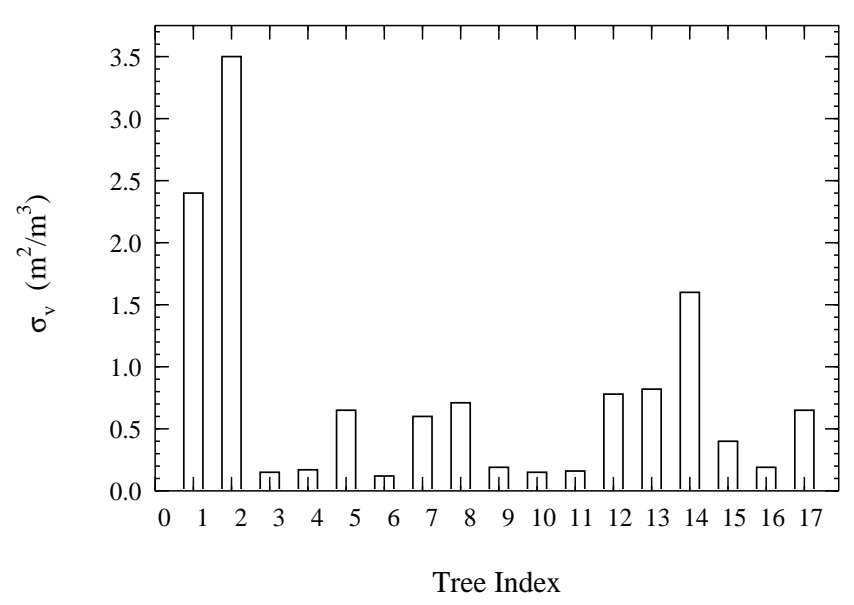

Figure 9: Dynamic range of the volume backscattering coefficients $\left(\mathrm{m}^{2} / \mathrm{m}^{3}\right)$ computed at $95 \mathrm{GHz}$ for the trees listed in Table 1.

points, the measured $\sigma_{v v}^{o}$ values are higher than the modeled $\sigma_{v v}^{o}$ values. This underestimation of the modeled $\sigma_{v v}^{o}$ values could be due to multiple scattering and/or non-uniformity in the canopy of these trees which are not accounted for in the model.

The effect of the presence of leaves, fruits or seed on the magnitude of the extinction coefficient is best demonstrated through multiple measurements of specific trees at different times of the year. In Fig. 12, the extinction coefficient of a Dogwood tree doubled in value when fruits became present in the canopy. In the case of a Dwarf 


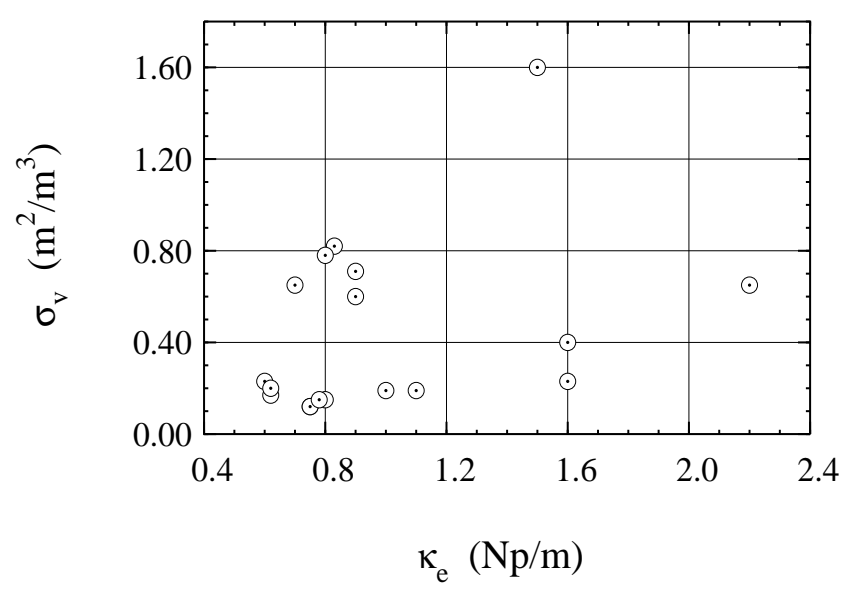

Figure 10: Correlation between the extinction coefficient and volume backscattering coefficient of 17 tree canopies measured at $95 \mathrm{GHz}$.

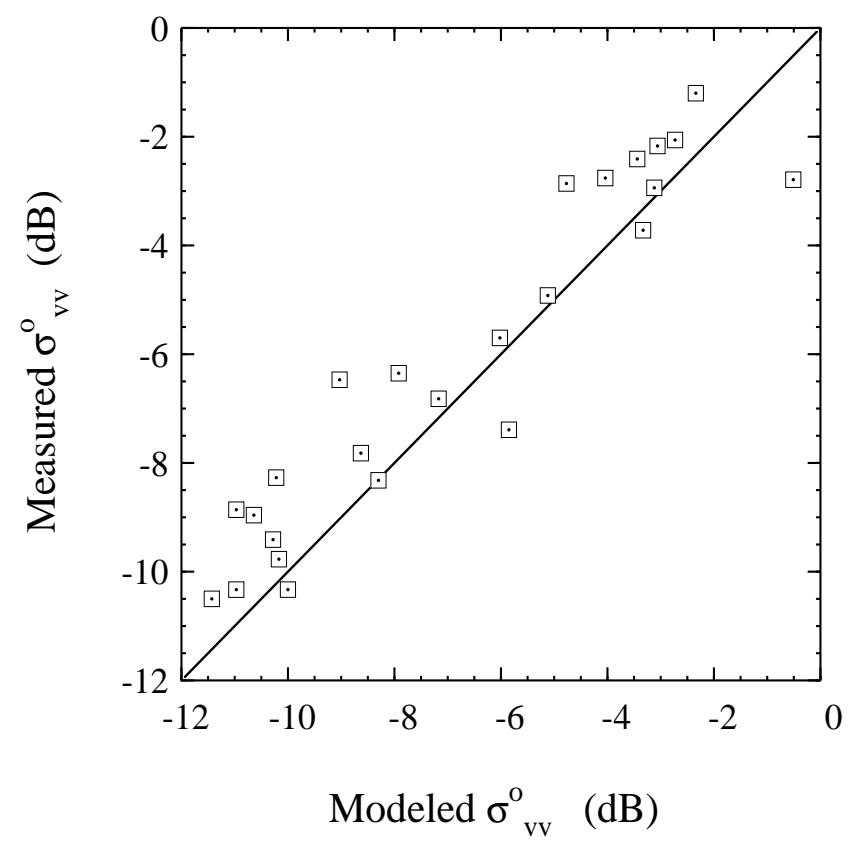

Figure 11: Comparison between measured $\sigma_{v v}^{o}$ and modeled $\sigma_{v v}^{o}$ for 17 tree canopies measured at $95 \mathrm{GHz}$.

Sumac tree, $\kappa_{e}$ was higher when fruits were present, as shown in Fig. 13. Furthermore, the extinction increased further in level with the further increase of fruits, even with the total loss of leaves. In contrast, in the example depicted in Fig. 14 for an Ash-Leaved Maple, the extinction coefficient appears to be dominated by the leaves. 


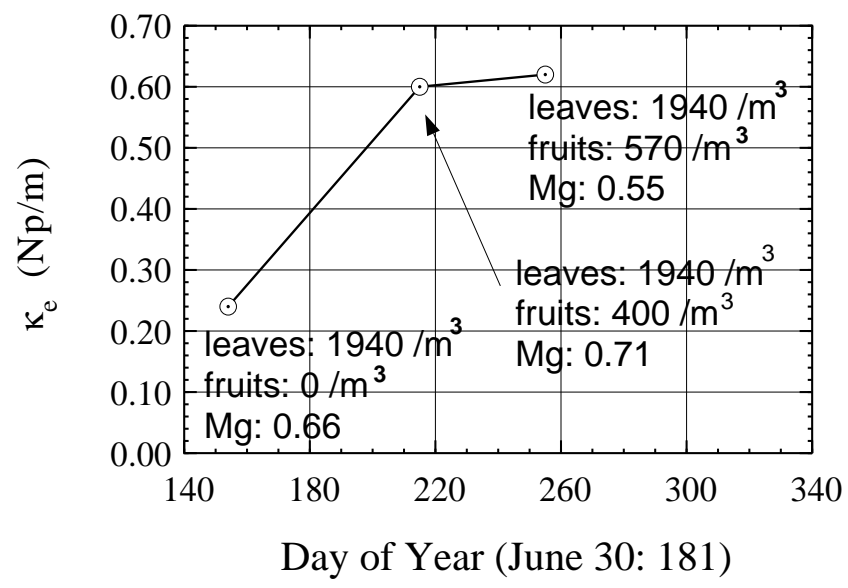

Figure 12: The extinction coefficient of a Dogwood tree canopy measured at $95 \mathrm{GHz}$ during Spring, Summer, and Fall.

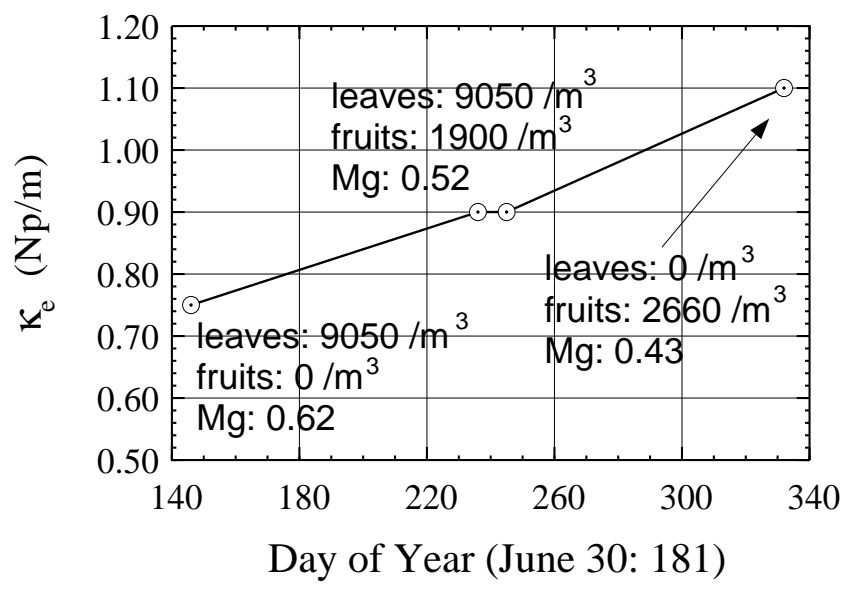

Figure 13: The extinction coefficient of a Dwarf Sumac tree canopy measured at $95 \mathrm{GHz}$ during Spring, Summer, and late Fall.

\section{CONCLUSIONS}

A new technique was developed for determining the extinction and volume backscattering coefficients in tree canopies using the measured radar backscatter time-domain response. The technique is simple and can be applied to already available wide-band radar backscatter data. After verifying the technique experimentally through a set of indoor measurements on an Arbor Vitae tree, it was used to determine the extinction and volume backscattering coefficients of 50 different tree canopies observed under various growth conditions. It 


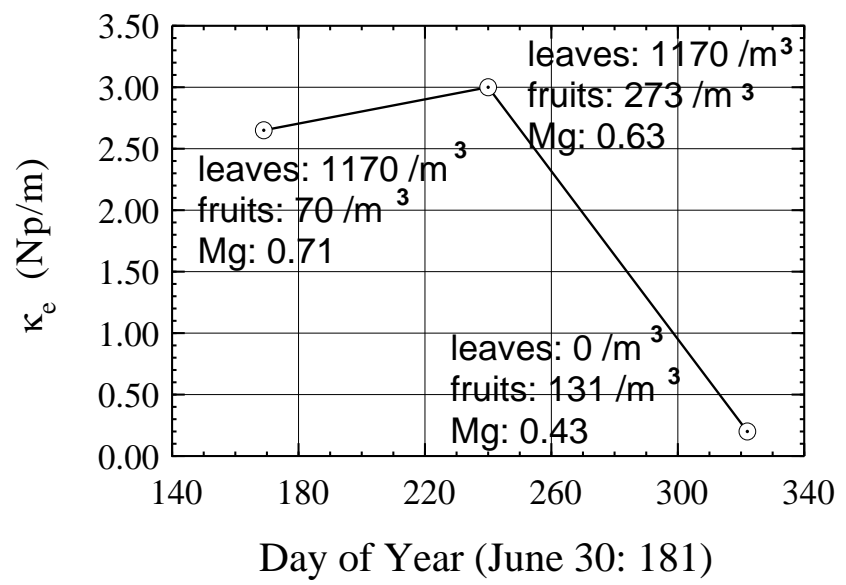

Figure 14: The extinction coefficient of an Ash-leaved Maple tree canopy measured at $95 \mathrm{GHz}$ during Spring, Summer, and late Fall.

was observed that extinction is slightly higher at $95 \mathrm{GHz}$ in comparison with that at $35 \mathrm{GHz}$, and that for the trees observed in this study, total attenuation through an individual tree exhibited a wide dynamic range extending from a low of $7 \mathrm{~dB}$ and a high of $60 \mathrm{~dB}$. In addition, extinction was found to be sensitive to the presence of fruits and to the leaf number density.

\section{REFERENCES}

[1] Borel, C. C. and R. E. McIntosh, "Millimeter Wave Backscatter from Deciduous Trees," IEEE Trans. Antennas and Propagat., vol. 38, no. 9, pp. 1391-1398, Sept. 1990.

[2] Narayanan, R. M., C. C. Borel, and R. E. McIntosh, "Radar Backscatter Characteristics of Trees at 215 GHz," IEEE Trans. Geosci. Remote Sensing, vol. 26, no. 3, pp. 217-228, May 1988.

[3] Mead, J. B., P. M. Langlois, P. S. Chang, and R. E. McIntosh, "Polarimetric Scattering from Natural Surfaces at 225 GHz," IEEE Trans. Antennas and Propagat., vol. 39, no. 9, pp. 1401-1411, Sept. 1991.

[4] Currie, N.C., F. B. Dyer, and E. E. Martin, "Millimeter Foliage Penetration Measurements," 1976 Int. IEEE Antennas and Propagat. Soc. Symp. Digest, Amherst, MA, 1976. 
[5] Violette, E. J., R. H. Espeland, and F. Schwering, "Vegetation Loss Measurements at 9.6, 28.8, and 57.6 GHz through a Pecan Orchard,” CECOM-83-2, U.S. Army Communications-Electronics Command, Fort Monmouth, NJ, March 1983.

[6] Schwering, F. K., E. J. Violette, and R. H. Espeland, "Millimeter-Wave Propagation in Vegetation: Experiments and Theory," IEEE Trans. Geosci. Remote Sensing, vol. 26, no. 3, pp. 355-367, May 1988.

[7] Ulaby, F. T., T. E. Van Deventer, J. R. East, T. F. Haddock, and M. E. Coluzzi, "Millimeter-Wave Bistatic Scattering From Ground and Vegetation Targets," IEEE Trans. Geosci. Remote Sensing, vol. 26, no. 3, pp. 229-243, May 1988.

[8] C. E. Mandt, Y. Kuga, L. Tsang, and A. Ishimaru,"Microwave Propagation and Scattering in a Dense Distribution of Non-tenuous Spheres: Experiment and Theory," Waves in Random Media, vol. 2, pp. 225234, 1992.

[9] M. T. Hallikainen, F. T. Ulaby, and M. Abdelrazik,"Dielectric Properties of Snow in the 3 to $37 \mathrm{GHz}$ Range," IEEE Trans. on Antennas and Propagat., vol. AP-34, no. 11, pp. 1329-1340, Nov. 1986.

[10] Ulaby, F.T. and C. Elachi Radar Polarimetry for Geoscience Applications, Artech House, Dedham MA, 1990.

[11] Ulaby, F.T., M.W. Whitt, and K. Sarabandi, “AVNA-based polarimetric scatterometers," IEEE AP magazine, vol. 32, 1990.

[12] Nashashibi, A., K. Sarabandi, and F.T. Ulaby,"A Calibration Technique For Polarimetric Coherent-OnReceive Radar Systems," IEEE Trans. Antennas and Propagat., vol. 43, no. 4, pp. 396-404, April 1995.

[13] F. T. Ulaby, R. K. Moore, and A. K. Fung,Microwave Remote Sensing: Active and Passive, Vol. II, Artech House, 1986. 


\begin{tabular}{||l|l|c|c|c|c|c|}
\hline In & Tree Name & Date & $\mathrm{Mg}$ & $n_{l}$ & $n_{f}$ & $\mathrm{~d}$ \\
\hline \hline 1 & Ashleaved Maple & $6 / 18$ & 0.71 & 1170 & 70 & 4.3 \\
\hline 2 & Ashleaved Maple & $8 / 28$ & 0.63 & 1170 & 273 & 4.3 \\
\hline \hline 3 & Norway Maple\#1 & $7 / 3$ & 0.61 & 180 & 0 & 10.5 \\
\hline \hline 4 & Norway Maple\#2 & $8 / 28$ & 0.53 & 443 & 0 & 7.3 \\
\hline \hline 5 & Dwarf Sumac\#1 & $8 / 24$ & 0.52 & 6250 & 900 & 2.4 \\
\hline \hline 6 & Dwarf Sumac\#2 & $5 / 26$ & 0.62 & 9048 & 1905 & 3.3 \\
\hline 7 & Dwarf Sumac\#2 & $8 / 24$ & 0.51 & 9048 & 400 & 3.3 \\
\hline 8 & Dwarf Sumac\#2 & $9 / 2$ & 0.53 & 8500 & 1150 & 3.3 \\
\hline 9 & Dwarf Sumac\#2 & $11 / 28$ & 0.43 & 0 & 2656 & 3.3 \\
\hline \hline 10 & Dogwood & $8 / 21$ & 0.57 & 2050 & 1370 & 4.5 \\
\hline \hline 11 & White Willow & $8 / 28$ & 0.62 & 1280 & 0 & 9.0 \\
\hline \hline 12 & Nannyberry & $9 / 2$ & 0.59 & 540 & 15 & 8.7 \\
\hline \hline 13 & Arbor Vitae\#1 & $5 / 21$ & & 3000 & 0 & 2.5 \\
\hline 14 & Arbor Vitae\#1 & $7 / 29$ & 0.57 & 3125 & 0 & 2.5 \\
\hline \hline 15 & Arbor Vitae\#2 & $9 / 12$ & 0.56 & 1436 & 1347 & 8.4 \\
\hline \hline 16 & White Spruce\#1 & $8 / 24$ & 0.59 & 1046 & 0 & 4.0 \\
\hline \hline 17 & White Spruce\#2 & $6 / 18$ & 0.65 & 1065 & 0 & 3.2 \\
\hline
\end{tabular}

where

In: Tree index,

Mg: gravimetric moisture content (g/g),

$n_{l}$ : number of leaves $/ \mathrm{m}^{3}$,

$n_{f}$ : number of fruits or seeds $/ \mathrm{m}^{3}$,

$\mathrm{d}$ : width of tree canopy in meters.

Table 1: Partial listing of physical parameters collected for several tree canopies. 